\title{
A novel TSC2 mutation in a patient with tuberous sclerosis complex
}

\author{
Minh Thu Tran $\mathrm{Vu}^{1}$, Thuy Vy Nguyen ${ }^{2}$, Bao Nam Tran Huynh ${ }^{3}$, Lan Anh Le ${ }^{3}$, Ngoc \\ Thieu Pham Nguyen ${ }^{3}$, Thi Dieu Ai Nguyen ${ }^{3}$, Ngoc Hong Phuong Le ${ }^{4}$, Vinh Pham \\ Nguyen $^{1}$, and Thuy Duong Ho Huynh ${ }^{2}$ \\ ${ }^{1}$ Tam Duc Heart Hospital \\ ${ }^{2}$ Vietnam National University Ho Chi Minh City University of Science \\ ${ }^{3}$ KTEST Science Company \\ ${ }^{4}$ Vietnam National University Ho Chi Minh City, School of Medicine
}

July 2, 2020

\begin{abstract}
We reported a patient presenting with tuberous sclerosis complex. Next-generation sequencing showing a frameshift mutation in TSC2 gene confirmed the clinical diagnosis. The study contributed to raise medical awareness on tuberous sclerosis complex in Vietnam that could lead to future approval of its diagnostics and treatment.
\end{abstract}

\section{KEYWORDS}

Tuberous sclerosis complex, frameshift mutation, TSC2 gene, next-generation sequencing

\section{Key Clinical Message}

We reported a patient presenting with tuberous sclerosis complex. Confirmatory diagnosis for the disorder by next-generation sequencing could contribute to induce awareness of health care personnel and patient's family about the disease.

\section{INTRODUCTION}

Tuberous sclerosis complex (TSC) is a rare autosomal dominant disorder characterized by the formation of hamartomas in multiple organs, most commonly in the brain, skin, heart, lungs, kidneys and eyes. ${ }^{1}$ Clinical manifestations and penetrance of the disease are highly variable and age-related, including epilepsy, mental disability, behavioral problems, angiofibromatosis, cardiac rhabdomyoma, renal angiomyolipoma, skin abnormalities. ${ }^{2,3}$ TSC is an inherited autosomal dominant disease with a high sporadic mutation rate, resulted from inactivating mutations in TSC 1 or TSC 2 tumor suppressor genes. TSC 2 encodes the $200-\mathrm{kDa}$ protein tuberin of which mutations usually cause a more severe clinical phenotype. ${ }^{3}$

\section{CASE REPORT}

A 17-year-old female patient was diagnosed as having heart tumor when she had a routine checkup in a local hospital and transferred to Tam Duc Heart Hospital was admitted to our hospital. The patient was born at full term, normal pregnancy and natural delivery, with no family history of epilepsy. At 5-month-old, the patient started suffering from convulsion after falling down from a hammock. She was then diagnosed with generalized epilepsy and prescribed anticonvulsant medication. From age 2, she experienced partial seizures with eight to ten episodes a year. Throughout the years, she was treated with anti-epileptic drugs including sodium valproate and carbamazepine without successful control of seizures. She stopped studying at age 15 due to intellectual disability. 
Physical examination showed facial angiofibromas over her nose, cheeks and forehead, intraoral fibromas and hypopigmented skin lesions (shagreen patch) on different parts of the body (Figure 1). Her biochemical laboratory test was unremarkable. Echocardiography showed chordae of tendinae sclerosis (Figure 2). Chordae tendineae sclerosis and the presence of lipofibroma in midlateral wall of left ventricle were also demonstrated by cardiac MRI (Figure 2). Cerebral MRI revealed multiple 5 to $7 \mathrm{~mm}$ nodules in the brain cortex and subependymal zone (Figure 3). Thoracic multi-slide computed tomography (MSCT) showed multiple 3-5 $\mathrm{mm}$ nodules in both lungs (Figure 4). Renal angiomyolipomas of $50 \times 50 \mathrm{~mm}$ in bilateral kidneys were revealed by ultrasonography and MSCT (Figure 5). With those characteristic clinical manifestations, we considered that the patient fulfilled diagnostic criteria of TSC.

Next generation sequencing of TSC 1 and TSC2 gene was performed on patient blood sample to confirm the diagnosis of TSC, after obtention of patient's parents signed informed consent. Library preparation was carried out using Trusight Cancer kit (Illumina, United States) according to the manufacturer's instructions. Prepared library was sequenced using Illumina Miniseq platform. Variants (SNPs and indels) were called using the Genome Analysis ToolKit (GATK) according to guidelines, and human reference genome hg38. ${ }^{4}$ Variants with at least 20X coverage were further analyzed using Alamut Visual (Interactive Biosoftware). Synonymous variants, intronic variants outside of the flanking regions, and variants with a minor allelic frequency (MAF) [?] $5 \%$ in the Genome Aggregation (gnomAD) database were excluded based on the American College of Medical Genetics and Genomics (ACMG) recommendations for variant frequency in control populations. ${ }^{5}$ The patient was found to carry a novel heterozygous frameshift mutation c.2626delA, p.Thr876ProfsTer18 (NM_000548.5) in exon 23 of TSC 2 gene. This mutation was classified as likely pathogenic based on ACMG guidelines. Sanger sequencing was used to confirm the identified mutation (Figure 6). This mutation was not identified in normal subjects according to gnomAD, and was not reported in HGMD, OMIM and Clinvar as associated with TSC.

There is no approved therapy protocol for TSC in Vietnam yet and the follow-up was not possible since the patient lives in distant region from our hospital.

\section{DISCUSSION}

The two tumor suppressor genes TSC 1 and TSC 2 were identified through their involvement in TSC with more than 1000 mutations localized throughout the genes. TSC 2 mutations accounted for approximately $70 \%$ of all disease-causing mutations and expressed more severe clinical phenotypes than TSC 1 mutations. ${ }^{3}$

The novel frameshift mutation found in this study was classified as likely pathogenic according to ACMG guidelines for variant classification. It was considered as member of the null variant group which includes nonsense, frameshift, canonical $+/-1$ or 2 splice sites, initiation codon, single or multi-exon deletion mutations. This is the most prominent disease-causing group found in TSC 2 gene. ${ }^{3}$ The mutation is located in the tuberin-type domain and was predicted to result in truncating and structural modification of the encoded protein. Different mutations were previously reported for TSC 2 gene. ${ }^{6-13}$ A novel frameshift mutation in exon 23 of TSC 2 gene classified as likely pathogenic was previously reported in Chinese patients suffering epilepsy. ${ }^{8}$ Another TSC 2 frameshift mutation was identified in a Bulgarian patient. ${ }^{10}$ Cases of lymphangiomyomatosis (LAM) considered as affecting almost exclusively young women resulted from mutations in the extreme carboxy-terminus of tuberin. ${ }^{11}$ However, the lymphangiomyomatosis phenotype observed in our patient was not due to mutation in Rap-Gap domain as previously described. The patient multisystemic impairments and severe clinical phenotypes were probably associated with the pathogenic variant identified in TSC 2 gene, but we cannot exclude the role of an additional unknown factor. Furthermore, the clear association of this novel mutation with the disease needs to be verified using cell and animal model for mutant protein function. We were not able to collect sample from the patient's family due to refusal to participate. Therefore, it was not possible to determine whether the mutation was sporadic or hereditary.

There was currently no available and approved therapy for TSC in Vietnam. Confirmatory diagnosis for TSC by next-generation sequencing could contribute to induce awareness of health care personnel and patient's family about the disease. 


\section{CONFLICT OF INTEREST}

The authors have no conflicts of interest to disclose.

\section{AUTHOR CONTRIBUTIONS}

MTTV: examined the patient, conducted clinical sampling and interpretation, patient counseling and drafted manuscript; TVN: carried out sequencing data analyses and drafted the manuscript; BNTH, LAL, and NTNP performed NGS, TDAN and NHPL performed Sanger sequencing; VPN: supervised clinical studies in Tam Duc Heart Hospital; TDHH: corresponding author, coordinated this study, and wrote the manuscript.

\section{REFERENCES}

1. Northrup H, Koenig MK, Pearson DA, et al. Tuberous Sclerosis Complex. In: Adam MP, Ardinger HH, Pagon RA, et al., eds. GeneReviews [Internet] . University of Washington, Seattle; 2020.

2. Northrup H, Krueger DA, Roberds S, et al. Tuberous sclerosis complex diagnostic criteria update: Recommendations of the 2012 international tuberous sclerosis complex consensus conference. Pediatr Neurol . 2013;49(4):243-254.

3. Salussolia CL, Klonowska K, Kwiatkowski DJ, Sahin M. Genetic Etiologies, Diagnosis, and Treatment of Tuberous Sclerosis Complex.Annu Rev Genomics Hum Genet . 2019;20(1):217-240.

4. Van der Auwera GA, Carneiro MO, Hartl C, et al. From FastQ Data to High-Confidence Variant Calls: The Genome Analysis Toolkit Best Practices Pipeline. In: Current Protocols in Bioinformatics . Vol 43. John Wiley \& Sons, Inc.; 2013:11.10.1-11.10.33.

5. Richards S, Aziz N, Bale S, et al. Standards and guidelines for the interpretation of sequence variants: a joint consensus recommendation of the American College of Medical Genetics and Genomics and the Association for Molecular Pathology. Genet Med . 2015;17(5):405-423.

6. Lee JS, Lim BC, Chae J-H, et al. Mutational analysis of paediatric patients with tuberous sclerosis complex in Korea: genotype and epilepsy. Epileptic Disord . 2014;16(4):449-455.

7. Rosset C, Vairo F, Bandeira IC, et al. Molecular analysis of TSC1 and TSC2 genes and phenotypic correlations in Brazilian families with tuberous sclerosis. PLoS One . 2017;12(10).

8. He J, Zhou W, Shi J, Lin J, Zhang B, Sun Z. TSC1 and TSC2 Gene Mutations in Chinese Tuberous Sclerosis Complex Patients Clinically Characterized by Epilepsy. Genet Test Mol Biomarkers . 2020;24(1):15 .

9. Gao S, Wang Z, Xie Y. Two novel TSC2 mutations in pediatric patientswith tuberous sclerosis complex: Case report. Med . 2018;97(29):e11533.

10. Glushkova M, Bojinova V, Koleva M, et al. Molecular genetic diagnostics of tuberous sclerosis complex in Bulgaria: six novel mutations in the TSC1 and TSC2 genes. J Genet . 2018;97(2):419-427.

11. Strizheva GD, Carsillo T, Kruger WD, Sullivan EJ, Ryu JH, Henske EP. The spectrum of mutations in TSC1 and TSC2 in women with tuberous sclerosis and lymphangiomyomatosis. Am J Respir Crit Care Med . 2001;163(1):253-258.

12. Zhang R, Wang J, Wang Q, et al. Identification of a novel TSC2 c.3610G > A, p.G1204R mutation contribute to aberrant splicing in a patient with classical tuberous sclerosis complex: A case report. $B M C$ Med Genet . 2018;19(1):1-8.

13. Giannantoni NM, Restuccia D, Della Marca G, Alfano RM, Vollono C. A novel TSC2 mutation causing tuberless tuberous sclerosis.Seizure . 2014;23(7):580-582.

\section{FIGURES LEGENDS}

FIGURE 1. Cutaneous manifestations of the proband. A, Facial angiofibromas; B, Shagreen patch. 
FIGURE 2. Echocardiography and cardiac magnetic resonance results of the proband. Chordae of tendinae sclerosis in parasternal long axis view (A) and in apical 4-chamber view (arrow). Lipofibroma $6 \times 22 \times 32$ $\mathrm{mm}$ in midlateral wall of left ventricle (C), (D) (arrow).

FIGURE 3. Cerebral magnetic imaging of the proband. Nodules $5-7 \mathrm{~mm}$ in brain cortex $(\mathrm{A}, \mathrm{B}, \mathrm{C})$ and subependymal zone (D).

FIGURE 4. Thoracic Computer Tomography results of the proband. Nodules $3-5 \mathrm{~mm}$ in lungs (green arrow).

FIGURE 5. Abdominal Computer Tomography results of the proband. Angiomyolipoma $50 \times 50 \mathrm{~mm}$ in two kidneys (green arrow).

FIGURE 6. A, Next generation sequencing revealed a c.2626delA (p.Thr876ProfsTer18) heterozygous mutation in the patient's TSC2 gene (framed by red box). B, Sanger sequencing confirmed the mutation found (red arrow).
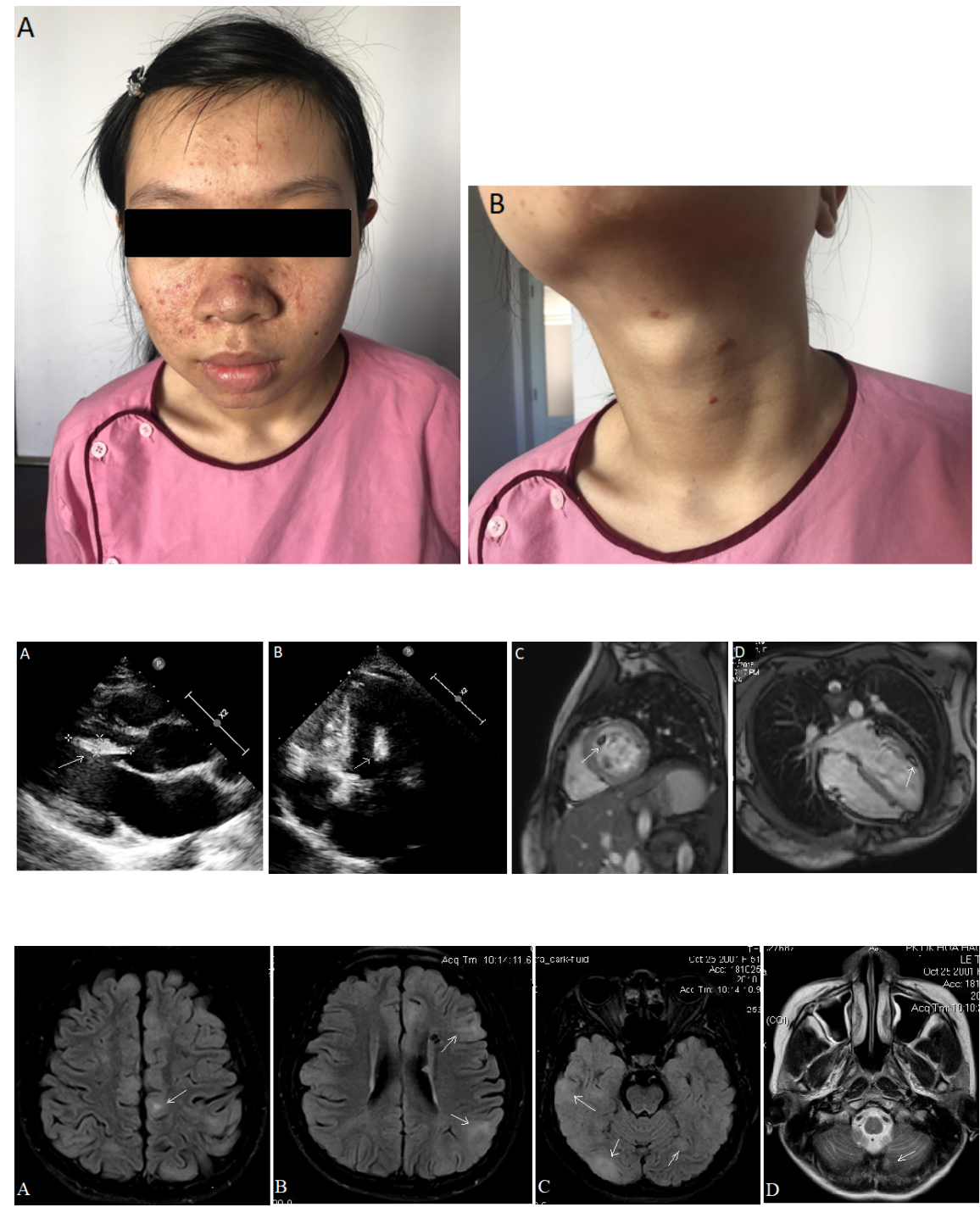

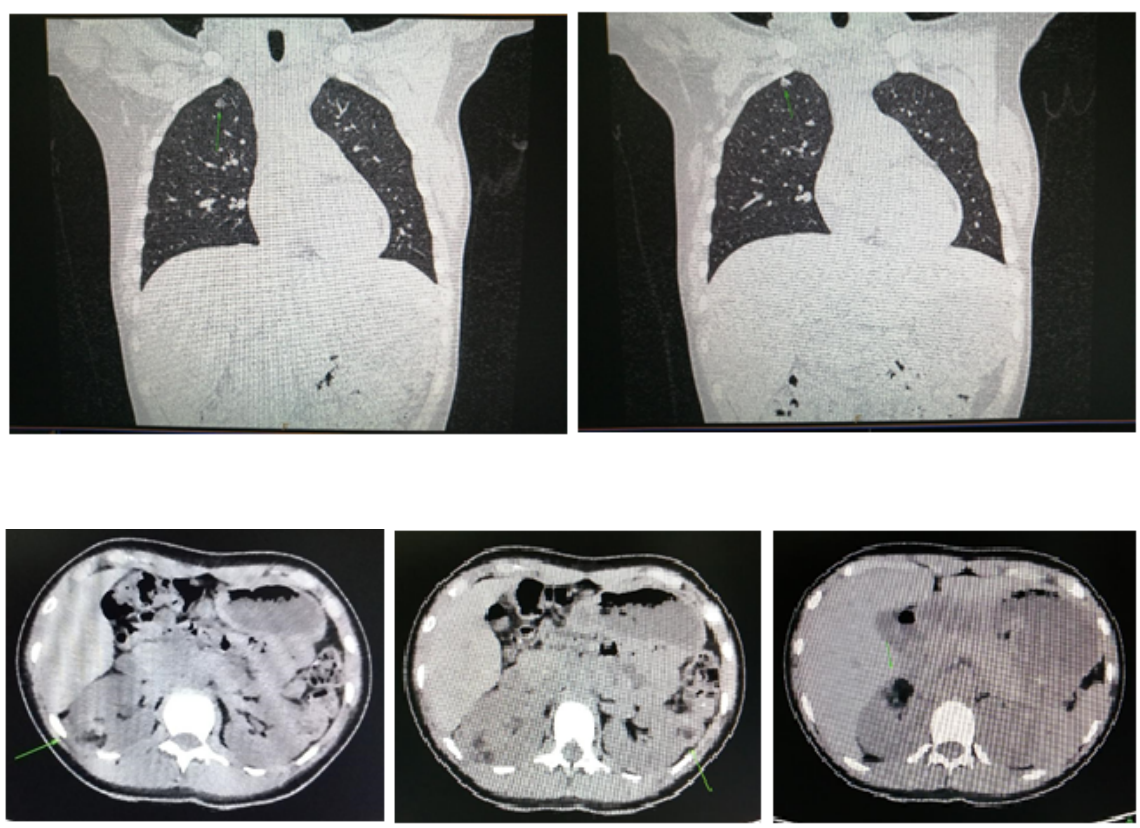

A
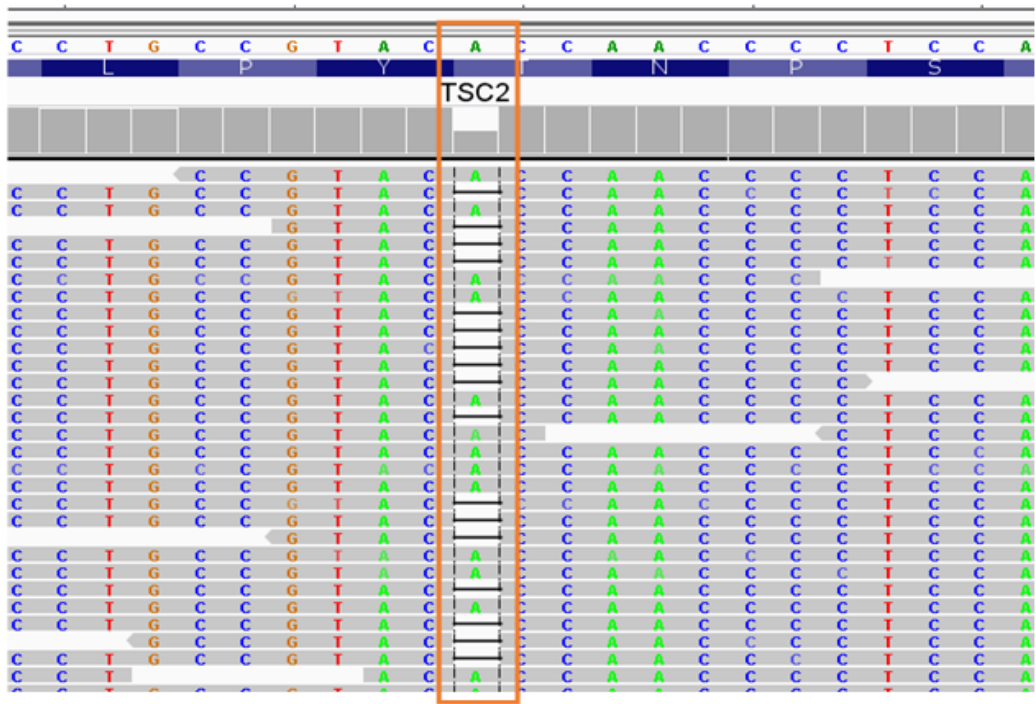

B

c. 2626delA

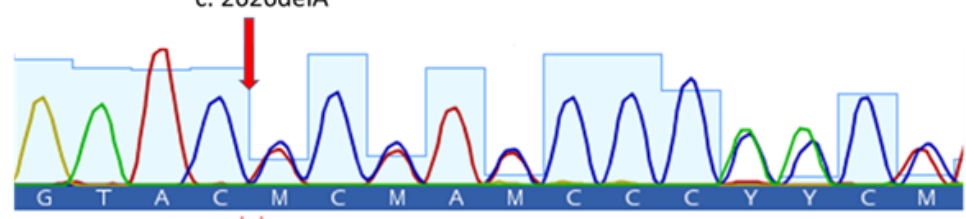

Normal control STACACCAACCCCTCCAAGT AAT GGTCCCCCAGCCCCT T 